\title{
RON and c-Met facilitate metastasis through the ERK signaling pathway in prostate cancer cells
}

\author{
BINBIN YIN ${ }^{1}$, ZHENPING LIU $^{1}$, YIYUN WANG ${ }^{1}$, XUCHU WANG ${ }^{2}$, WEIWEI LIU ${ }^{2}$, \\ PAN YU ${ }^{2}$, XIUZHI DUAN ${ }^{2}$, CHUNHUA LIU ${ }^{3}$, YUHUA CHEN $^{2}$, YURONG ZHANG $^{2}$, \\ XIAOYAN PAN ${ }^{2}$, HANGPING YAO $^{4}$, ZHAOPING LIAO $^{3}$ and ZHIHUA TAO ${ }^{1,2}$

\begin{abstract}
${ }^{1}$ Laboratory of Medical Genetics, College of Laboratory Medicine and Life Sciences, Wenzhou Medical University, Wenzhou, Zhejiang; Departments of ${ }^{2}$ Laboratory Medicine and ${ }^{3}$ Blood Transfusion, The Second Affiliated Hospital of Zhejiang University School of Medicine, Hangzhou, Zhejiang; ${ }^{4}$ Key Laboratory of Laboratory Medicine,
\end{abstract} \\ Chinese Ministry of Education, Zhejiang Provincial Key State Key Laboratory for Diagnosis and Treatment of Infectious \\ Diseases, First Affiliated Hospital of Zhejiang University School of Medicine Hangzhou, Hangzhou, Zhejiang, P.R. China
}

Received September 24, 2016; Accepted April 5, 2017

DOI: $10.3892 /$ or.2017.5585

\begin{abstract}
Prostate cancer (PCa) is a metastatic malignant cancer driven by complex pathological mechanisms and characterized by poor long-term prognosis. Metastasis is the main cause of death of PCa patients, yet the molecular mechanisms of this process are poorly understood. In the present study, positive co-expression of RON and c-Met was observed in human clinical PCa tissues (biopsy material), as detected by immunohistochemical staining and quantitative real-time PCR. We investigated this further in PCa cells, demonstrating that the inhibition of RON and c-Met with foretinib (GSK1363089) suppressed metastasis and promoted the reversal of the epithelial-to-mesenchymal transition (EMT) in PCa cells. Furthermore, the invasion and migration of $\mathrm{PCa}$ cells were enhanced by the exogenous activation of RON with MSP and c-Met with HGF, whereas silencing of RON and c-Met attenuated the invasion and metastasis of the PCa cells. Our data also demonstrated that HGF/c-Met, but not the MSP-RON signaling pathway may be the dominant mechanism for PCa EMT. We further revealed that RON and c-Met facilitate metastasis via ERK1/2 signaling. These findings indicate that RON and c-Met facilitate metastasis through ERK1/2 signaling and that targeting RON and c-Met with foretinib may be an attractive therapeutic option for suppressing PCa metastasis.
\end{abstract}

Correspondence to: Dr Zhaoping Liao, Department of Blood Transfusion, The Second Affiliated Hospital of Zhejiang University School of Medicine, Hangzhou, Zhejiang 310009, P.R. China E-mail: jjlzp@126.com

Professor Zhihua Tao, Laboratory of Medical Genetics, College of Laboratory Medicine and Life Sciences, Wenzhou Medical University, Wenzhou, Zhejiang 325035, P.R. China

E-mail: zrtzh@zju.edu.cn

Key words: RON, c-Met, metastasis, prostate cancer, ERK, foretinib

\section{Introduction}

In the United States, prostate cancer (PCa) has become the most commonly diagnosed male cancer and the second leading cause of cancer-related deaths, with an estimated 220,800 annual diagnoses and 27,540 annual deaths during 2015 (1). With increasing incidence and mortality, PCa is a current global health issue. Although many advanced therapies have significantly improved the survival of PCa patients, the number of deaths is still substantial. Current treatments are not effective once cancer metastasis occurs, as there are no therapies that cure metastatic disease or prevent the metastatic process. Epithelial-to-mesenchymal transition (EMT) is a key link in the induction of pathological changes in cancer cells, which plays a central role in cancer development and progression. Carcinoma cells undergo EMT which is a reversible phenotypic change in which epithelial cells lose cell-cell adhesion and epithelial polarization and are thus converted to a mesenchymal phenotype, gaining increased motility and invasiveness (3). In PCa, the EMT may contribute to metastasis and intravasation from the organ of origin to distant sites. Therefore, in order to more successfully treat $\mathrm{PCa}$, a deeper understanding of the biological mechanisms that are involved in regulating PCa metastasis is necessary.

The RON and c-Met receptor are $\sim 180-\mathrm{kDa}$ heterodimeric proteins composed of a $35-\mathrm{kDa} \alpha$-chain and a $145-\mathrm{kDa} \beta$-chain linked by disulfide bonds that share $60 \%$ similarity in their functional domains $(4,5)$. RON and c-Met are the only members of the MET proto-oncogene family expressed in humans, and they play important roles during embryonic development, but are mostly dormant after birth. However, both receptors are activated once tissue becomes cancerous. RON and c-Met are activated by their corresponding ligands [macrophage stimulating protein (MSP) for RON and hepatocyte growth factor (HGF) for c-Met]. RON was firstly identified as a member of the MET subfamily by Ronsin and colleagues in 1993 (6). Shortly afterwards, other researchers found that MSP acts as a specific ligand for RON. c-Met was first discovered in 
1984 as an oncogene and its ligand HGF was identified in 1991 (7). The MSP/RON and HGF/c-Met signaling pathways have gained increasing attention in the fields of tumor biology and therapy over the past 20 years. They have been found to be necessary for the survival and growth of cancer cells in many tumors (8). Patients with $\mathrm{RON}^{+} / \mathrm{c}-\mathrm{Met}^{+}$tumors have significantly poorer 10 -year disease-free survival rates than those with RON-/c-Met tumors (11.8 vs. $79.3 \%$, respectively; $\mathrm{P}=0.009$ ) (9). Thus, RON/c-Met expression has potential as a prognostic biomarker for cancer progression in some cancers, and it is crucial to understand the mechanism of RON/c-Met signaling in PCa.

Foretinib is a high affinity multikinase of the tyrosine kinase inhibitor (TKI) family that inhibits c-Met at a halfmaximal inhibitory concentration $\left(\mathrm{IC}_{50}\right)$ of $0.4 \mathrm{nM}$ and $\mathrm{RON}$ at an $\mathrm{IC}_{50}$ of $3 \mathrm{nM}$. It has been demonstrated that foretinib has antitumor activity in different tumor types (10-13) and phase II clinical trials with foretinib have been initiated to investigate its ability to treat diverse cancers, including head and neck, renal, glioblastoma, gastric and liver cancers (14-18). To date, however, the ability of foretinib to treat $\mathrm{PCa}$ is still unknown.

In the present study, we identified a markedly positive co-expression of RON and c-Met in human PCa tissues by immunohistochemical staining and quantitative real-time PCR. We demonstrated that targeting RON and c-Met using foretinib (GSK1363089) significantly suppressed metastasis and promoted the reversal of EMT in PCa cells. Additionally, we further revealed that RON and c-Met facilitate metastasis via ERK1/2 signaling.

\section{Materials and methods}

Cell culture. PCa cell lines (PC3, DU145, 22RV1 and LNCaP) were purchased from the Chinese Academy of Sciences Cell Bank (Shanghai, China). All the cell lines were maintained in RPMI-1640 medium supplemented with $10 \%$ fetal bovine serum (FBS; Gibco, Carlsbad, CA, USA) and 1\% penicillin/ streptomycin (Jinuo Biomedical Technology, Co., Ltd., Hangzhou, China), in a $5 \% \mathrm{CO}_{2}$ atmosphere at $37^{\circ} \mathrm{C}$. Treatment with foretinib (Shanghai Selleck Chemicals, Co., Ltd., Shanghai, China), recombinant human HGF (Thermal Tech) and recombinant human MSP (R\&D Systems, Minneapolis, MN, USA) was performed according to the manufacturer's instructions.

Clinical samples and immunohistochemical staining. Ten pairs of PCa and their corresponding peri-cancer tissues were obtained from patients at The Second Affiliated Hospital of Zhejiang University School of Medicine (Hangzhou, China). Ethical approval was obtained from the Second Affiliated Hospital of Zhejiang University School of Medicine Research Ethics Committee, and written informed consent was obtained from all patients. PCa tissue samples were used for quantitative reverse transcription PCR (qRT-PCR) and sectioned at $5-\mu \mathrm{m}$ sections for IHC. The slides were incubated with human anti-RON (1:100), c-Met (1:200) and secondary antibodies, and the sections were developed in diaminobenzidine solution under a microscope and counterstained with hematoxylin. Negative control slides omitting the primary antibodies were included in all assays.
Western blot analysis. Western blot analysis was performed to detect the levels of RON (1:5,000) (a gift from Professor Hangping Yao, Zhejiang University, Hangzhou, China), c-Met (1:1,000; Cell Signaling Technology, Danvers, MA, USA), E-cadherin (1:2,000; Cell Signaling Technology), N-cadherin (1:1,000; Ruiying Biological, Suzhou, China), AR (1:2,000; Cell Signaling Technology), p-ERK1/2 (1:1,000; Cell Signaling Technology), ERK1/2 (1:1,000; Cell Signaling Technology) and GAPDH (1:5,000; Proteintech Group Inc., Chicago, IL, USA). Cell lysates were extracted with RIPA buffer (Beyotime Institute of Biotechnology, Shanghai, China) with proteinase inhibitors (Shanghai Selleck Chemicals) and a cocktail of phosphatase inhibitors (Shanghai Selleck Chemicals). Protein samples were separated by SDS-PAGE and transferred to an PVDF membrane. After blocking the non-specific binding for $2 \mathrm{~h}$ with $5 \%$ non-fat milk, the membranes were incubated overnight at $4^{\circ} \mathrm{C}$ with the specific primary antibodies. On the following day, the membranes were further incubated with HRP-conjugated secondary antibodies for $2 \mathrm{~h}$ at room temperature and recorded with the ChemiDoc XRS System (Bio-Rad Laboratories, Hercules, CA, USA).

Quantitative real-time PCR. Total RNA was isolated from tumor sample or cells using TRIzol reagent (Takara Bio, Dalian, China) and reversely transcribed using the PrimeScript RT-PCR kit (Takara Bio) according to the protocol. qRT-PCR was performed on the ABI 7900 Prism HT (Applied Biosystems, Foster City, CA, USA), followed by melting curve analysis. The $2^{-\Delta \Delta \mathrm{Ct}}$ method was used to assess the gene expression levels. Primers used are as follows: RON forward, CTT TGACGTGAAGTACGTGGT and reverse, CGTATGGCTA CAAACACAGCAC; c-Met forward, AGCGTCAACAGA GGGACCT and reverse, AGCGTCAACAGAGGGACCT; GAPDH forward, GCACCGTCAAGGCTGAGAAC and reverse, TGGTGAAGACGCCAGTGGA.

Immunofluorescence. Cells were cultured in a 24-well plate, and then were fixed with $4 \%$ paraformaldehyde for $20 \mathrm{~min}$, permeabilized with $0.1 \%$ Triton $\mathrm{X}-100$ for $15 \mathrm{~min}$ and blocked with $5 \%$ bovine serum albumin (BSA; Sharp, Solon, OH, USA). The samples were incubated with primary antibody at $4^{\circ} \mathrm{C}$ overnight, and then were incubated with DyLight 594 conjugated goat anti-rabbit IgG (1:200; Abbkine, Inc., Redlands, CA, USA) at room temperature for $2 \mathrm{~h}$. Nuclei were stained with DAPI (Beyotime Institute of Biotechnology) for $15 \mathrm{~min}$. Following a final rinse of three times with phosphate-buffered saline (PBS), the cells were imaged using inverted fluorescent microscope (Leica Microsystems, Wetzlar, Germany).

Migration and invasion assays. A 24-well Transwell plate was used to assess the migration and invasion of the PCa cells. Briefly, for the Transwell migration assay, $5 \times 10^{4}$ cells in $200 \mu \mathrm{l}$ serum-free RPMI-1640 were seeded on the upper chamber, $800 \mu \mathrm{l}$ of medium containing 5\% FBS was placed in the lower chamber of each well and incubated at $37^{\circ} \mathrm{C}$ for $24 \mathrm{~h}$. The cell invasion assay was carried out similarly, except that Matrigel (BD Biosciences, San Jose, CA, USA) was added to each well according to the manufacturer's instructions before cells were seeded on the upper chamber. After the time of incubation, non-migrated or non-invaded cells were gently removed from 
the upper chamber with a cotton swab. The cells in the lower chamber were fixed using $4 \%$ paraformaldehyde and stained with $0.1 \%$ crystal violet. The cells that were located on the underside of the filter were counted in 5 randomly selected fields.

Wound healing assay. Wound healing assay was conducted to examine the capacity of cell migration. Briefly, a wound was generated when the cells reached $80-90 \%$ confluency in a 6 -well plate by scratching the surface with a $1,000-\mu 1$ pipette tip. The cells were then incubated in $1 \%$ FBS for $24 \mathrm{~h}$, and then photographed using phase contrast microscopy (Leica Microsystems). The distance between the wound edges of the scratch area was analyzed using Adobe Photoshop 7.0. All experiments were performed in triplicate.

Small interfering RNA transfection. Small interfering RNA (siRNA) duplexes targeting RON and c-Met genes (Shanghai GenePharma, Co., Ltd., Shanghai, China) were transfected into PCa cells using Lipofectamine 2000 reagent (Invitrogen, Carlsbad, CA, USA) according to the manufacturer's instructions: siRNA duplexes targeting RON (forward, 5'-CCUG CUGGACACACUAAUUTT-3' and reverse, 5'-AAUUAGUG UGUCCAGCAGGTT-3'); c-Met (forward, 5'-GCAACAGCU GAAUCUGCAATT-3' and reverse, 5'-GCAACAGCUGAAU CUGCAATT-3'); and scramble siRNA duplex (forward, 5'-UUCUCCGAACGUGUCACGUTT-3' and reverse, 5'-ACG UGACACGUUCGGAGAATT-3'). The silencing effect was measured by western blotting and qRT-PCR $48 \mathrm{~h}$ post-transfection.

Cell scattering. Cells were plated in a 6-well plate at a density of 500 cells/well and allowed to form colonies for 7 days. Cell colonies were incubated with MSP and HGF for $24 \mathrm{~h}$ and then photographed. This protocol was according to a previous study (19).

Statistical analysis. Statistical calculations and graphical presentation were performed using GraphPad Prism 5.0. Data are expressed as mean and SD, unless otherwise indicated. Continuous variables were evaluated using an unpaired Student's t-test for comparisons between two groups. Statistical significance was confirmed at $\mathrm{P}<0.05$.

\section{Results}

Robust RON and c-Met expression in PCa. RON and c-Met expression has been assessed in a variety of types of cancer, yet there has been no study concerning the co-expression of RON and c-Met in PCa. To determine the expression of RON and c-Met, we first analyzed RON and c-Met in PCa tissues and non-cancerous prostate tissues by IHC. As shown in Fig. 1A, high RON and c-Met expression was observed in the $\mathrm{PCa}$ tissues and only minimal expression was noted in the non-cancerous prostate tissues. Moreover, a positive correlation was observed between the relative expression levels of RON and c-Met mRNA in the prostate tissues (Fig. 1B). To further explore the role of RON and c-Met in $\mathrm{PCa}$, we evaluated the expression of RON and c-Met in PCa cell lines. As shown via western blotting, qRT-PCR and immunofluorescent staining, RON and c-Met were highly expressed in the PC3 and DU145 cells, but exhibited little to no expression in the 22RV1 and LNCaP cells (Fig. 1C and D). In contrast, 22RV1 and LNCaP cells displayed high expression of AR, while PC3 and DU145 cells exhibited negligible expression of this protein (data not shown). Further studies of the migration and invasive potential of PCa cell lines revealed that PC3 and DU145 cells, which have high RON and c-Met expression, showed relatively higher migration and invasion abilities when compared to the 22RV1 and LNCaP cells, two low RON and c-Met-expressing prostate cancer cell lines (Fig. 1E). Therefore, the expression of RON and c-Met was found to be positively correlated with the metastatic potential of the PCa cell lines.

Inhibition of RON and c-Met using foretinib suppresses $P C a$ cell migration and invasion. To further investigate the role of RON and c-Met in PCa, we next determined whether RON and c-Met could be therapeutic targets for PCa. PC3 and DU145 cells were exposed to the RON and c-Met inhibitor foretinib (GSK1363089) for $24 \mathrm{~h}$. The effect of foretinib on RON and c-Met expression was confirmed by western blot analysis (Fig. 2A). Foretinib (20 nM) downregulated RON and c-Met expression in the cells, while also leading to increased E-cadherin and decreased $\mathrm{N}$-cadherin expression (Fig. 2A). E-cadherin is a canonical epithelial cell marker, while $\mathrm{N}$-cadherin is canonically associated with mesenchymal cells, suggesting that foretinib treatment reversed the EMT in these cells. In contrast, these responses to foretinib were not observed in the 22RV1 and LNCaP cells (data not shown). The migratory and invasive potentials of PC3 and DU145 cells were also extremely impaired after treatment with foretinib $(20 \mathrm{nM})$. As shown in Fig. 2B-D, foretinib (20 nM) significantly impaired both the wound closure ability and invasive potential of the PC3 and DU145 cells. These data suggest that RON and c-Met mediate PCa metastasis, and foretinib may have potential as a therapeutic agent for $\mathrm{PCa}$ metastasis.

RON enhances cell migration and invasion of PCa cells. We next investigated whether the knockdown of RON by siRNA would phenocopy the functional effects of foretinib treatment and promote the reversal of EMT by examining the expression of characteristic epithelial and mesenchymal cell markers. Transfection of the RON-targeting siRNA in the PC3 and DU145 cells almost completely abrogated RON protein expression, and led to a $60-70 \%$ decrease in RON mRNA expression (Fig. 3A). Remarkably, the knockdown of RON did not substantially alter E-cadherin and N-cadherin expression compared with the control groups (Fig. 3B). Migration and invasion assays were carried out to evaluate the effects of RON knockdown on cell migratory and invasive capacity. Our data revealed that the knockdown of RON attenuated the cell migratory and invasive abilities of the PC3 and DU145 cells (Figs. 3C and D and 5A). Furthermore, in order to analyze the function of RON, we exogenously activated RON with recombinant human MSP (100 ng/ml) for $24 \mathrm{~h}$. After treatment with MSP, the invasive and migratory abilities of the PC3 and DU145 cells were significantly increased (Figs. 3C and D and 5A). Treatment with MSP did not substantially alter E-cadherin and $\mathrm{N}$-cadherin expression (Fig. 3B), nor did it cause the cells 
A
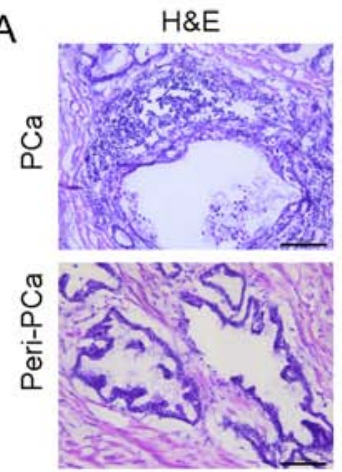

C
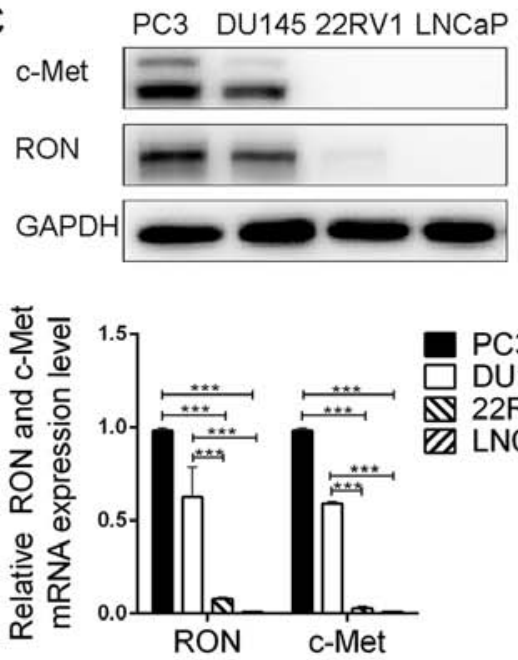

c-Met

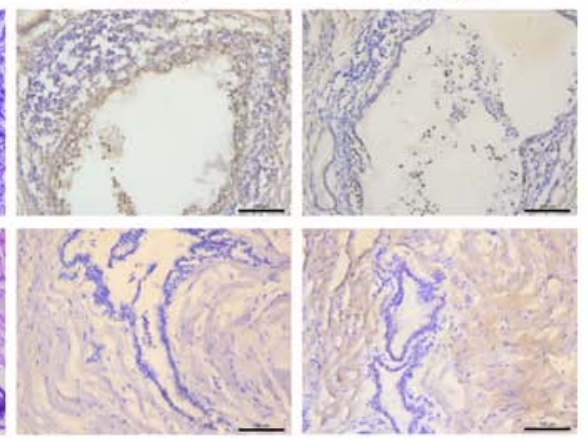

PC3

DU145

22RV1

$\square$ LNCaP
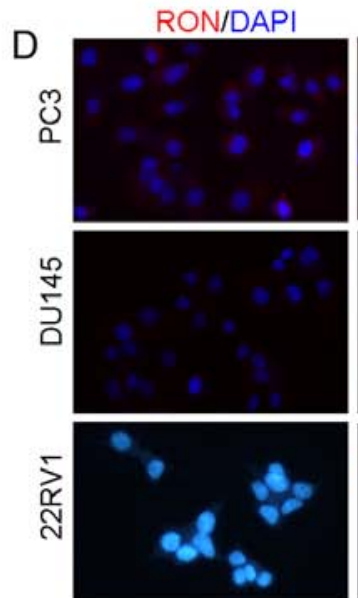

B
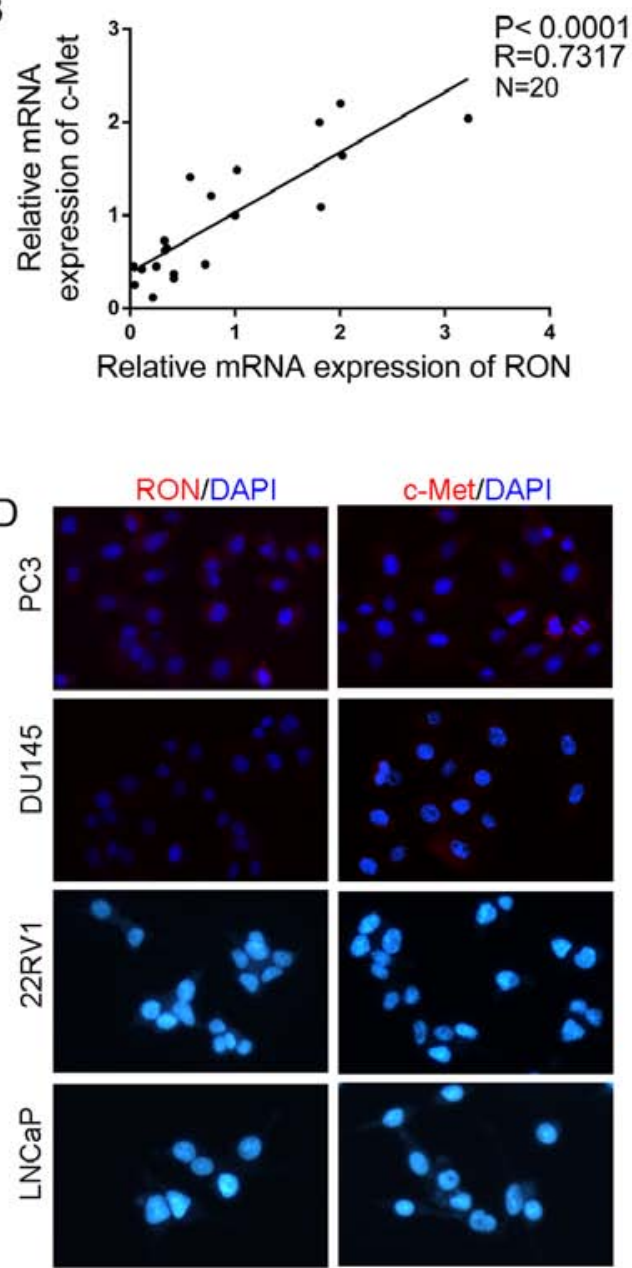
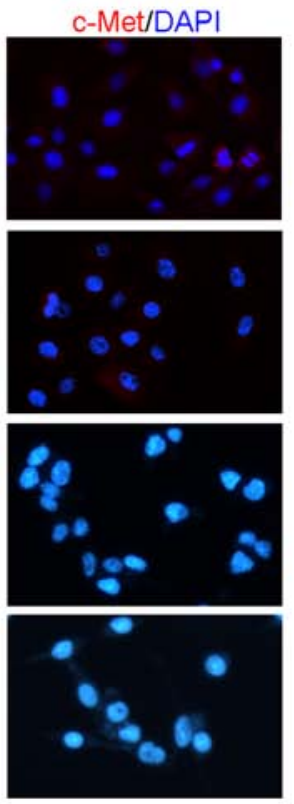
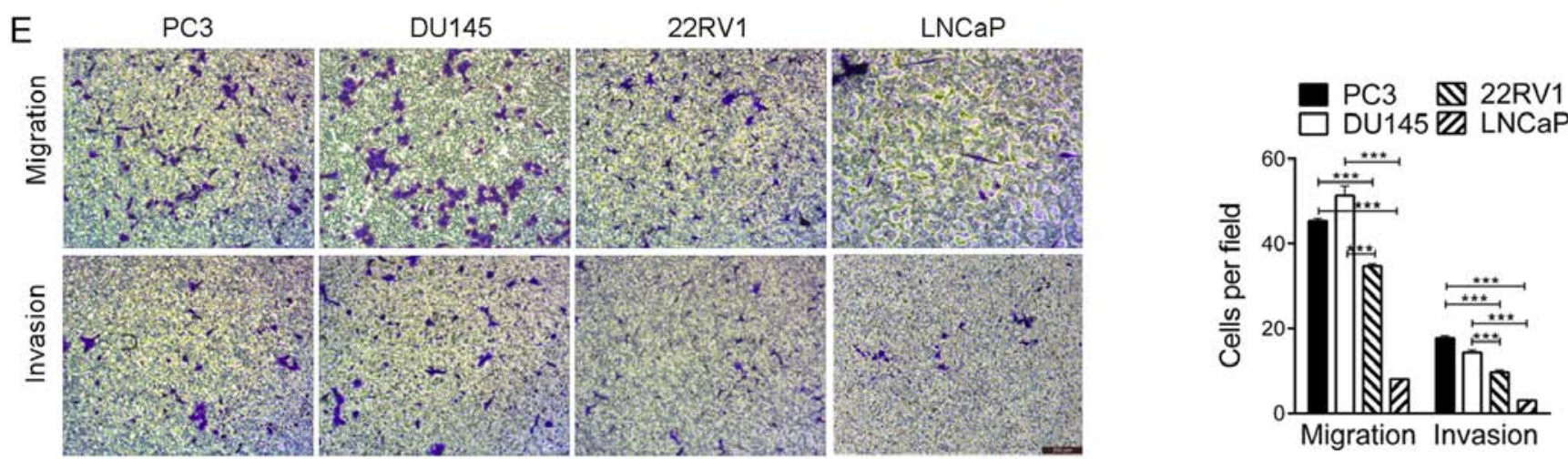

Figure 1. RON and c-Met are overexpressed in prostate cancer tissues and are associated with metastasis in PCa cell lines. (A) H\&E staining and immunohistochemistry using antibodies against RON and c-Met in normal prostate tissues and their corresponding prostate cancerous tissues (magnification, $\mathrm{x} 100$ ). (B) Correlation between RON and c-Met mRNA expression levels in clinical specimens. Each mRNA level is relative to that of GAPDH. The Pearson correlation test and linear regression were used to determine the relationship between the two genes tested. There was a significant correlation between RON and c-Met $(\mathrm{P}<0.0001, \mathrm{R}=0.7317, \mathrm{~N}=20)$. ( $\mathrm{C}$ and $\mathrm{D}) \mathrm{RON}$ and c-Met expression were examined by western blot analysis, RT-PCR and immunofluorescent analysis. (E) The metastatic potential of PCa cells was analyzed by migration and invasion assays (magnification, $\mathrm{x} 200)$. ${ }^{* * *} \mathrm{P}<0.001$.

to adopt a spindle-shaped mesenchymal morphology (data not shown).

c-Met enhances cell migration and invasion in PCa. To further understand the function of c-Met in PCa progression, we decreased the expression of c-Met in the PC3 and DU145 cells via siRNA-mediated knockdown. We confirmed the efficiency of the siRNA by western blot analysis and qRT-PCR, and observed almost complete loss of c-Met protein expression and a $60-70 \%$ decrease in c-Met mRNA expression in both the PC3 and DU145 cells (Fig. 4A). We next examined the expression of characteristic epithelial and mesenchymal markers. Remarkably, the knockdown of c-Met increased E-cadherin and reduced N-cadherin expression in the PC3 and DU145 cells compared to these levels in the control groups (Fig. 4B). Migration and invasion assays were utilized to determine the 
A
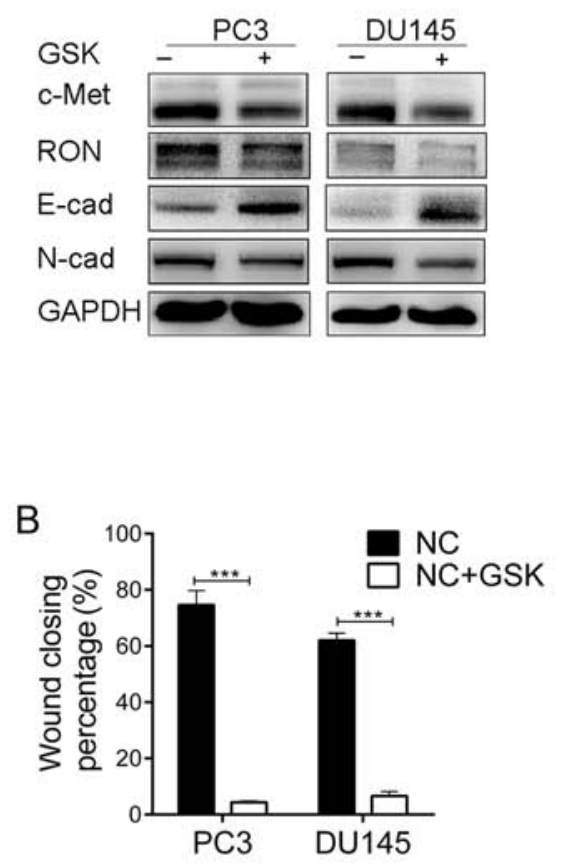
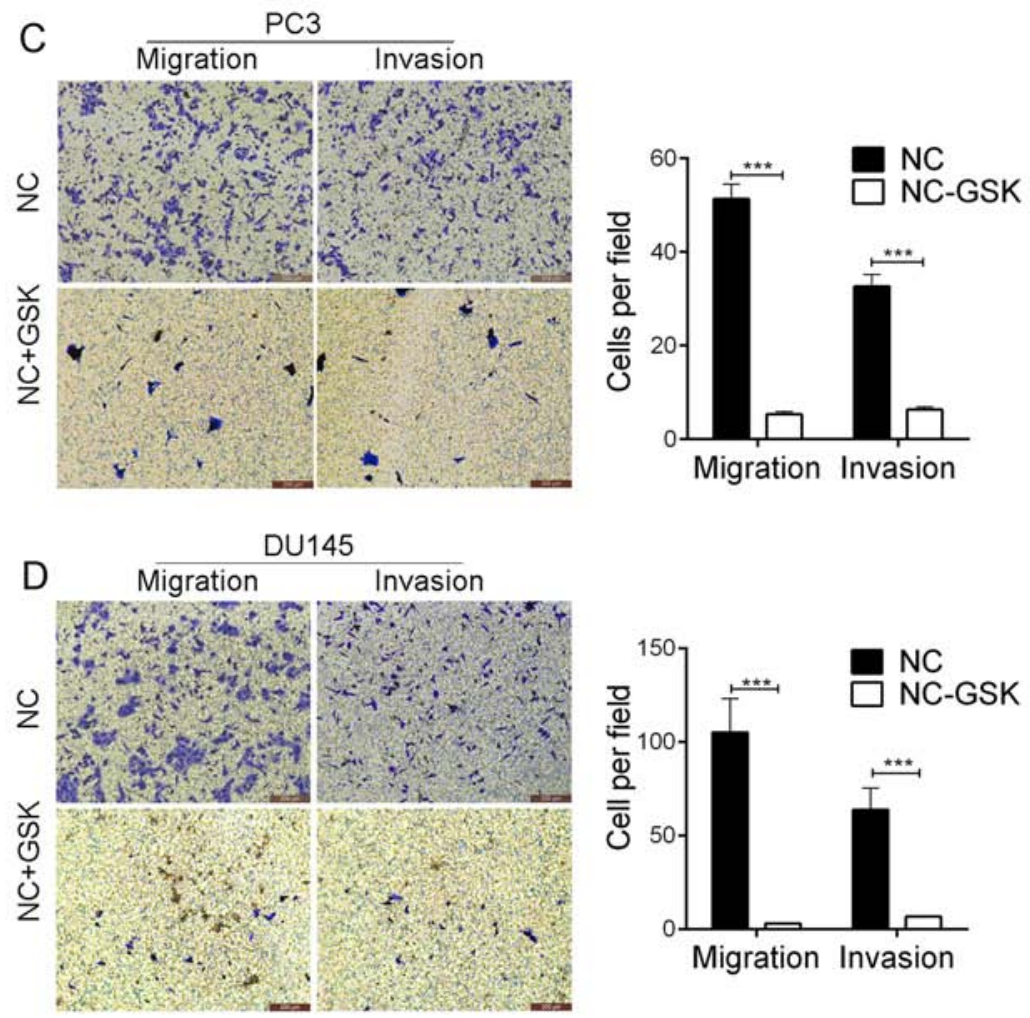

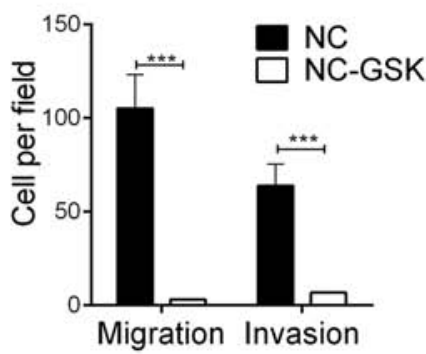

Figure 2. Inhibition of RON and c-Met using foretinib (GSK) inhibits the migration and invasion of PCa cell lines. (A) PC3 and DU145 cells were treated with foretinib $(20 \mathrm{nM})$ for $24 \mathrm{~h}$. Whole-cell lysates from indicated cells were analyzed by western blot analysis for RON, c-Met, E-cadherin, N-cadherin and GAPDH. (B) Wound healing assay was performed to determine the effect of foretinib (20 nM) on the migration of PC3 and DU145 cells. (C and D) PC3 and DU145 cells were treated with foretinib $(20 \mathrm{nM})$ for $24 \mathrm{~h}$. Migratory and invasive behaviors were analyzed by migration and invasion assays (magnification, $\mathrm{x} 200){ }^{* * * *} \mathrm{P}<0.001$.

A

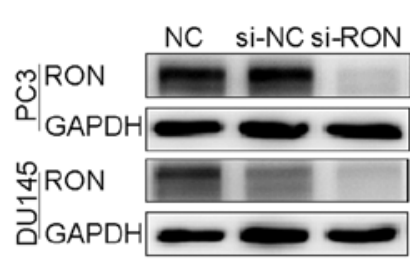

B

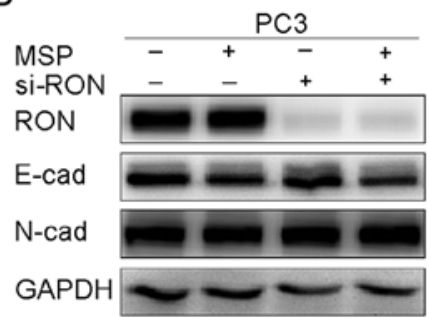

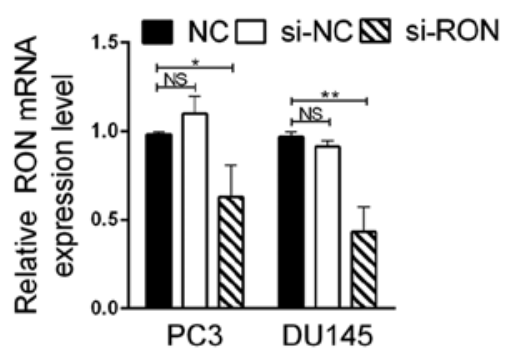

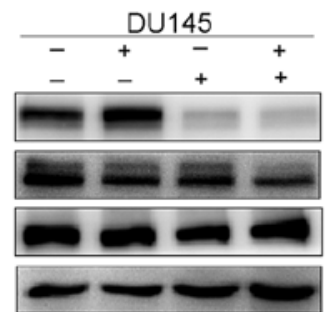

C

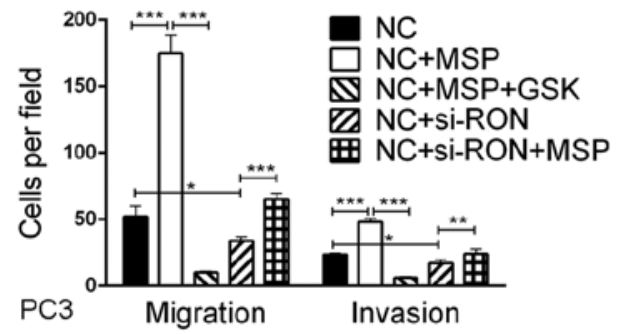

D

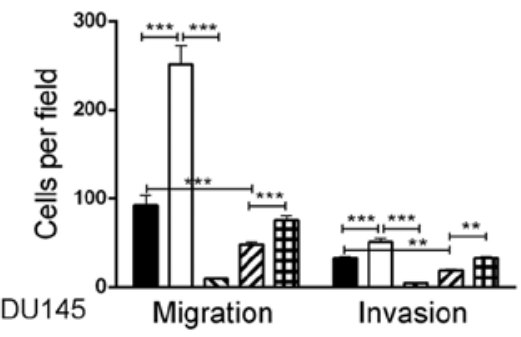

Figure 3. Functional role of RON in the migration and invasion of PCa cell lines. (A) Detection of siRNA-mediated knockdown of RON in PC3 and DU145 cells by western blotting and real-time PCR. (B) The expression of RON, E-cadherin and N-cadherin was determined by western blotting after RON knockdown and MSP (100 ng/ml) stimulation in PC3 and DU145 cells. (C and D) Transwell assays for the effects of RON silencing and MSP (100 ng/ml) on the migratory and invasive potentials of PC 3 and DU145 cells. ${ }^{*} \mathrm{P}<0.05,{ }^{* *} \mathrm{P}<0.01,{ }^{* * *} \mathrm{P}<0.001$; NS, not significant.

effects of c-Met on the migratory and invasive capacities of cells. The results showed that knockdown of c-Met expression resulted in significant suppression of the migration and inva- sion of both PC3 and DU145 cell lines (Figs. 4C and D and 5B). Following treatment with HGF, the invasive and migratory abilities of the PC3 and DU145 cells were significantly 
A

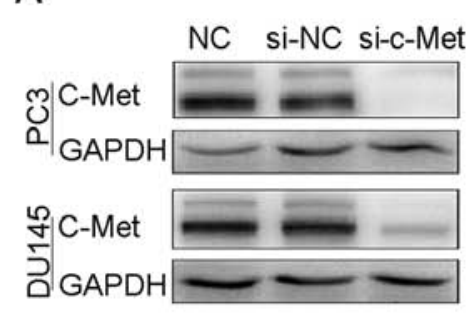

B

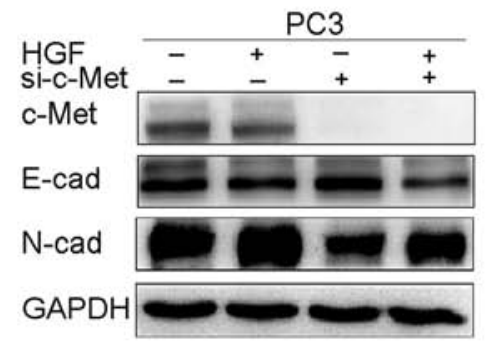

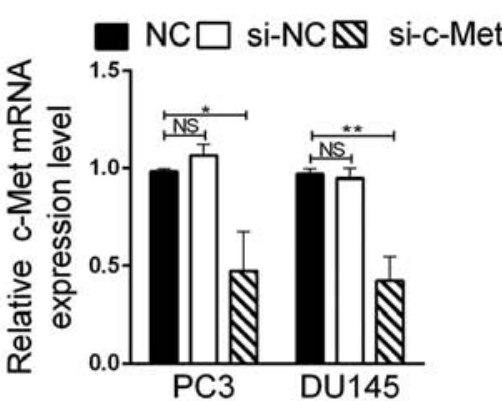

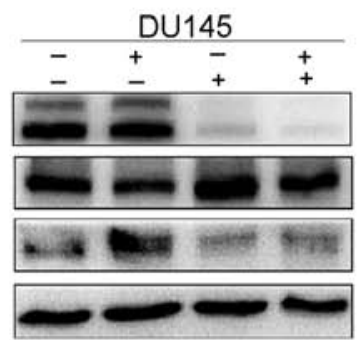

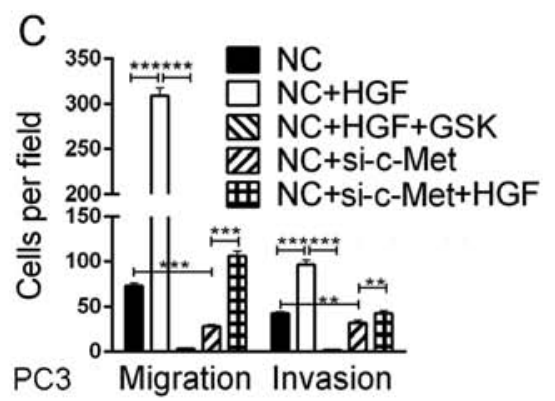

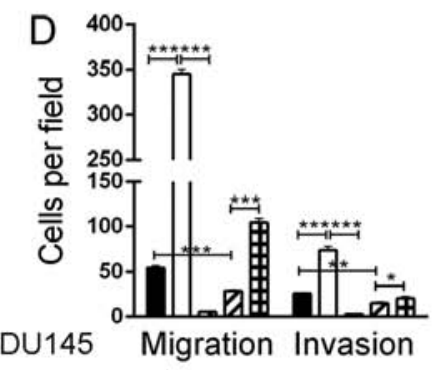

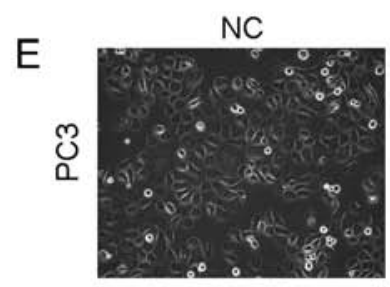
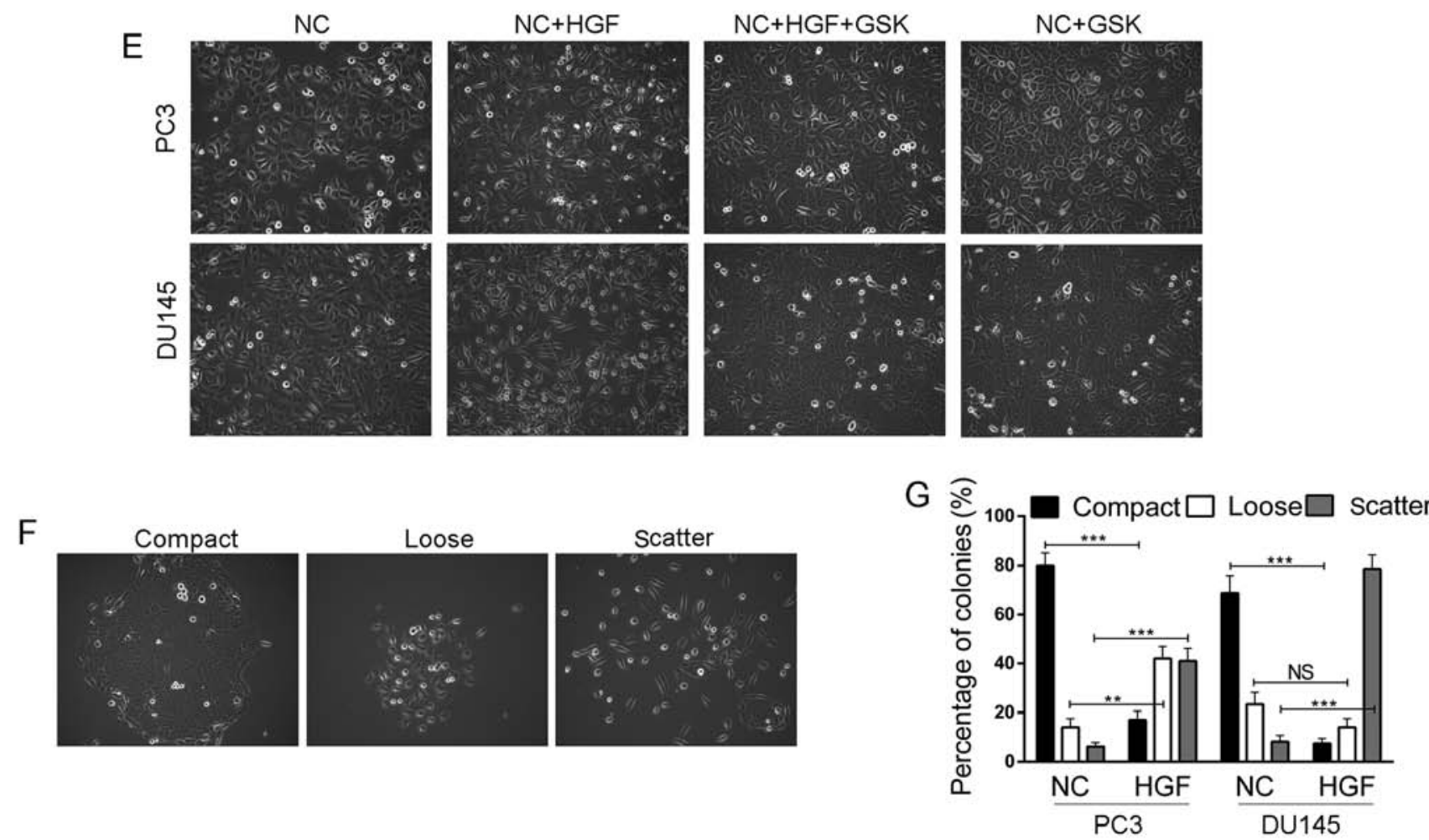

Figure 4. Functional role of c-Met in the metastasis and invasion of PCa cell lines. (A) Detection of the siRNA-mediated knockdown of c-Met in PC3 and DU145 cells by real-time PCR and western blotting. (B) The expression of c-Met, E-cadherin and N-cadherin was determined by western blotting after c-Met knockdown and HGF $(50 \mathrm{ng} / \mathrm{ml})$ stimulation in the PC3 and DU145 cells. (C and D) Transwell assays of the effects of c-Met silencing and HGF (50 ng/ml) on the migratory and invasive potentials of the PC 3 and DU145 cells. (E) Morphological changes of PC 3 and DU145 cells stimulated by HGF (50 ng/ml) and foretinib. (F and G) HGF (50 ng/ml) induced cell scattering in the PC3 and DU145 cells. ${ }^{*} \mathrm{P}<0.05,{ }^{* *} \mathrm{P}<0.01,{ }^{* * * *} \mathrm{P}<0.001$; NS, not significant.

increased (Figs. 4C and D and 5B). In addition, treatment with HGF induced cell scattering and caused the cells to adopt a spindle-shaped mesenchymal morphology (Fig. 4E), accompanied by increased expression of $\mathrm{N}$-cadherin and decreased expression of E-cadherin (Fig. 4B). In contrast, treatment with MSP had no effect (Fig. 3B). Moreover, foretinib could inhibit these in vitro changes in morphology, invasiveness and migratory potential caused by HGF in PCa cells (Fig. 4C and $\mathrm{E}$ and $5 \mathrm{~B})$. These results indicate that the HGF/c-Met signaling pathway may be a dominant factor in PCa cell EMT and play an important role in regulating $\mathrm{PCa}$ cell migration and invasion.

The ERK1/2 pathway mediates RON and c-Met-induced PCa metastasis. A previous study revealed that $\mathrm{RON}$ and c-Met are 
A

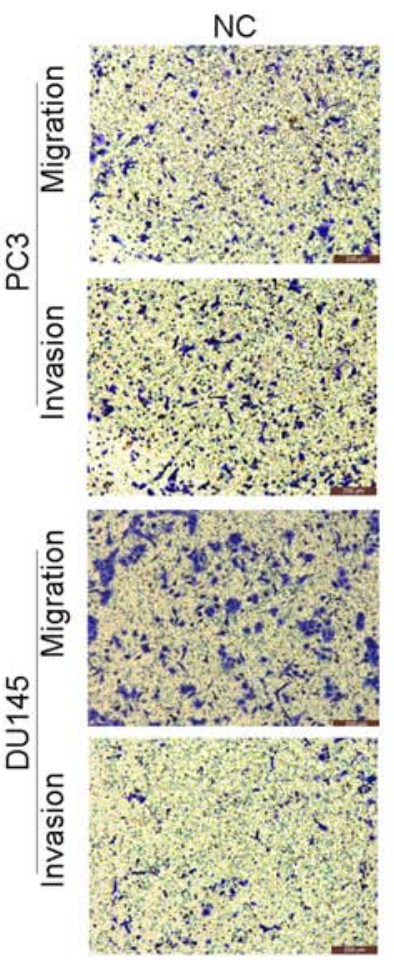

B
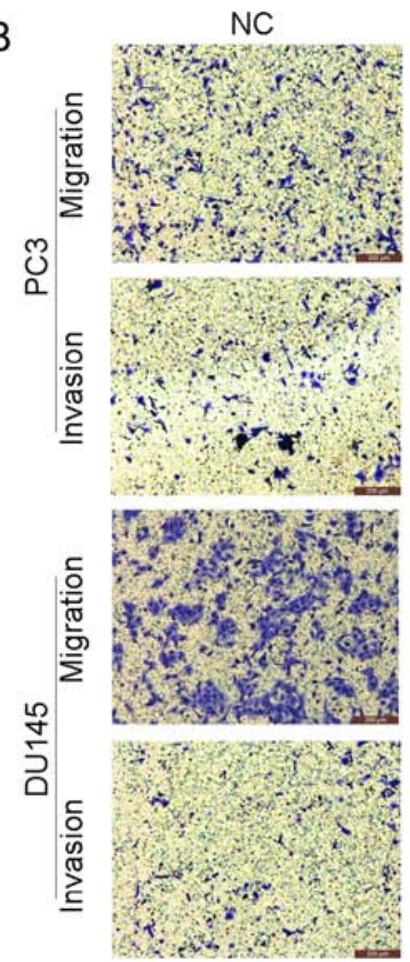

NC+MSP
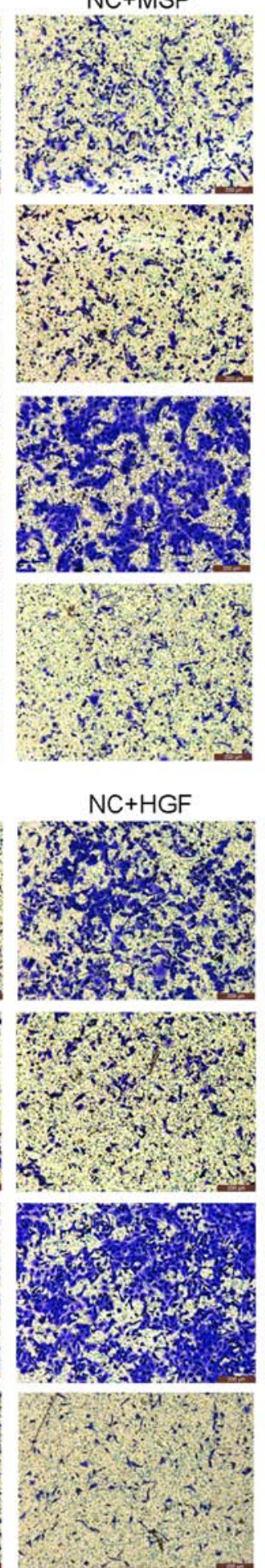
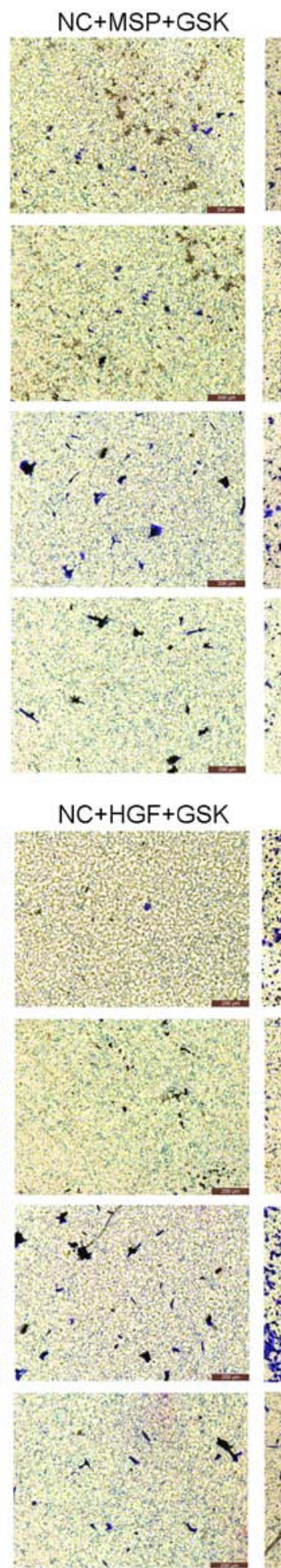
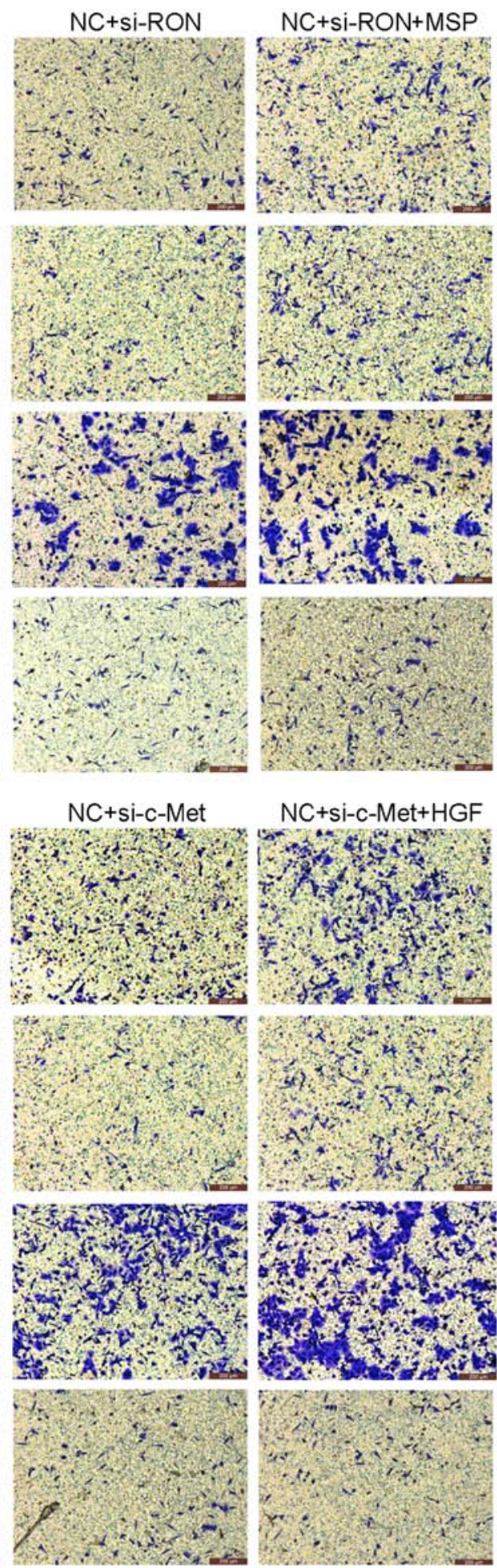

Figure 5. Functional role of RON and c-Met in the metastasis and invasion of PCa cell lines. (A) Transwell assays for the effects of si-RON and MSP (100 ng/ $\mathrm{ml}$ ) on migratory and invasive potentials of PC3 and DU145 cells (magnification, x200). (B) Transwell assays for the effects of si-c-Met and HGF (50 ng/ml) on the migratory and invasive potentials of PC 3 and DU145 cells (magnification, x200).

canonically mediated by ERK1/2 signaling (19). Therefore, we verified whether this signaling pathway could be responsible for the induction of RON and c-Met that mediates PCa metastasis. Our results demonstrated that the levels of p-ERK $1 / 2$ were suppressed by the siRNA-mediated knockdown of RON and c-Met and significantly increased following activation by MSP or HGF (Fig. 6A and B), compared with the control cells.
In addition, PC3 and DU145 cells were pre-incubated with U0126, a highly selective inhibitor of the ERK1/2 pathway and its upstream kinase. Blockade of the ERK1/2 pathway by U0126 significantly suppressed the MSP- or HGF-induced migration and invasion of the PC 3 and DU145 cells (Fig. 6C and D). These findings suggest that the ERK1/2 pathway may play a critical role in RON- and c-Met-induced metastasis. 
A

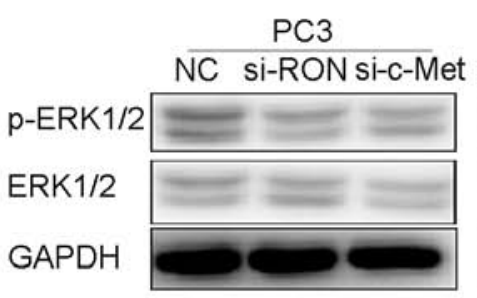

DU145

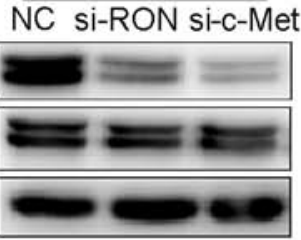

B

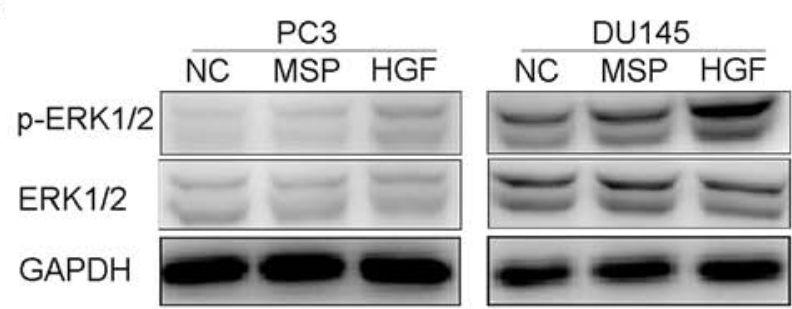

C

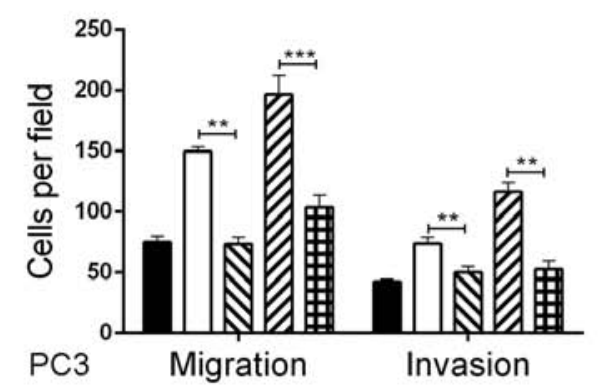

NC

$\square$ NC-MSP

NC-MSP-U0126

$\mathbb{Z} \mathrm{NC}-\mathrm{HGF}$

NC-HGF-U0126

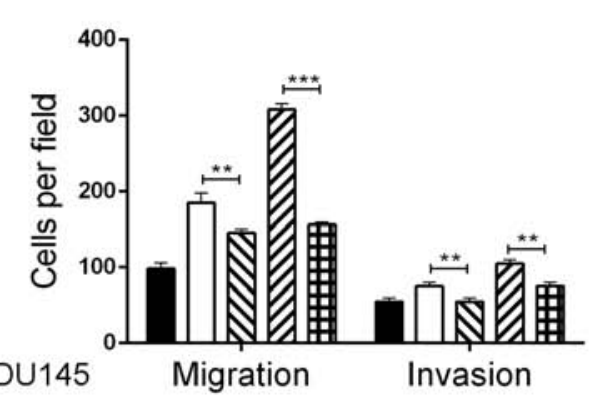

D
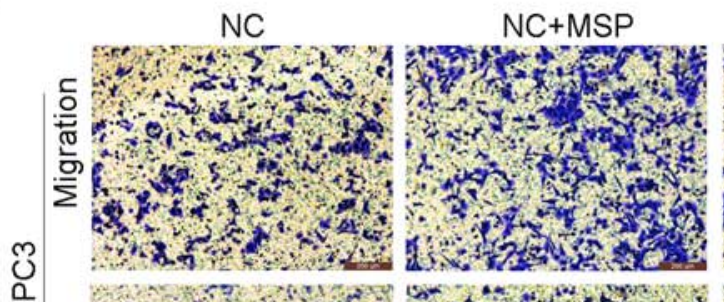

$\mathrm{NC}+\mathrm{MSP}+\mathrm{U} 0126$
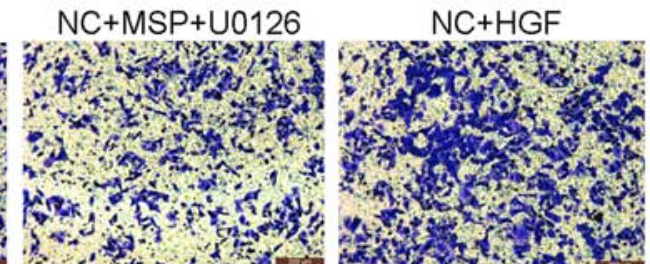

$\mathrm{NC}+\mathrm{HGF}+\mathrm{U} 0126$

8
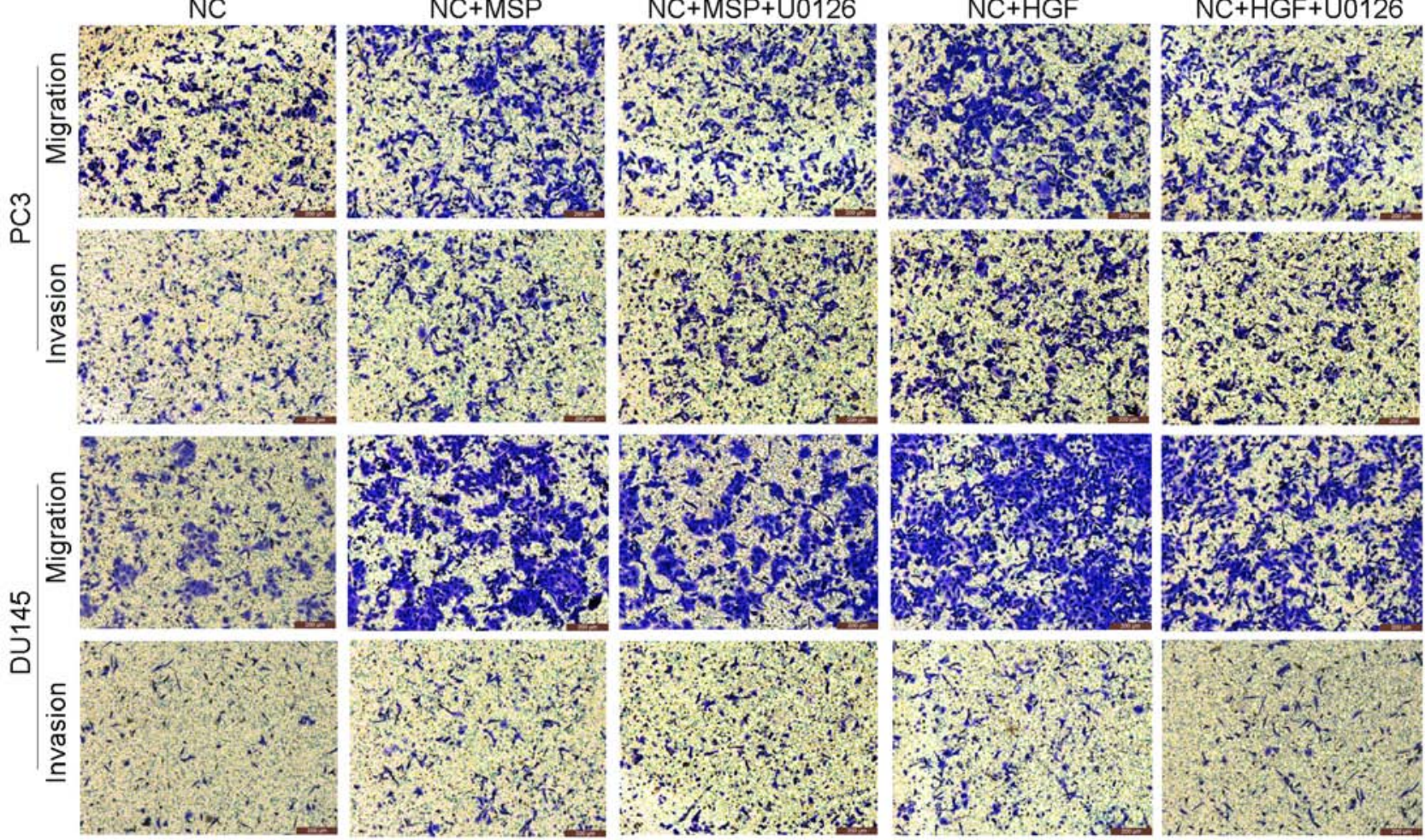

Figure 6. RON and c-Met mediates PCa metastasis via the ERK1/2 pathway. (A) Effect of RON and c-Met on phosphorylation of p-ERK1/2 was analyzed by western blotting. (B) PC3 and DU145 cells were treated with MSP or HGF for $24 \mathrm{~h}$. Level of p-ERK1/2 was detected by western blotting. (C and D) Migration and invasion assays were carried out in the PC3 and DU145 cells following treatment with MSP, HGF and U0126 for 24 h (magnification, x200). Each experiment was performed in triplicate. ${ }^{* *} \mathrm{P}<0.01,{ }^{* * *} \mathrm{P}<0.001$.

\section{Discussion}

PCa is a male malignancy of global health concern deserving intensive investigation. Metastasis is the leading cause of the poor prognosis of PCa patients. Although substantial progress has been made over the past decade towards uncovering the molecular mechanism underlying PCa metastasis, it remains incompletely understood. A better understanding of $\mathrm{PCa}$ metastasis could contribute to more efficient treatments.
The high expression of RON or c-Met has been reported in many tumors, including gastric, prostate, ovarian and breast cancer, and has been identified as a marker for poor prognosis (21). Moreover, a previous study reported that co-expression of RON and c-Met in node-negative breast cancer patients appears to result in an aggressive phenotype compared with a disease-free group (9). In the present study, we showed increased RON and c-Met expression in PCa and found that the expression of RON mRNA was 
positively correlated with c-Met mRNA in prostate tissues (Fig. 1A and B). Although we did not find a relationship between RON and c-Met expression with PCa grade severity in this limited sample size, our results suggest that the co-expression of RON and c-Met in prostate tissue may play a key role in PCa progression. Furthermore, we found that RON and c-Met were robustly expressed in highly metastatic PCa cell lines (PC3 and DU145), but barely expressed in lowmetastatic cell lines (22RV1 and LNCaP) (Fig. 1C-E). Such findings suggest that RON and c-Met play a vital role in $\mathrm{PCa}$ metastasis and help to identify suitable cell lines for the study of pathogenic mechanism of RON and c-Met in PCa. Thus, RON and c-Met may be used as biomarkers to predict cancer progression (20).

Although RON and c-Met share $60 \%$ structural similarity and are co-expressed in various human cancers contributing to tumor progression, the functions of RON and c-Met have not been fully elucidated $(22,23)$. Due to the similar structure between RON and c-Met, TKIs that are specific to either RON or c-Met have not yet been reported, with most TKIs having dual-specific effects on both RON and c-Met (20). Previous studies have supported the concept that RON/c-Met inhibition might represent a promising therapeutic option for suppressing the metastatic progression of cancer. Therefore, we analyzed the effects of RON and c-Met suppression on PCa invasive and migratory ability using the RON and c-Met dual inhibitor foretinib (Fig. 2B-D). Our data showed that foretinib significantly reduced metastasis and implies that RON and c-Met may be potential therapeutic targets for PCa metastasis. However, the cellular and molecular mechanism of RON and c-Met in cancer must be fully investigated in further studies to achieve maximal efficacy of TKIs.

As a proof of concept, we knocked down the expression of RON and c-Met or activated RON and c-Met in PCa cells. As expected, we found that silencing of RON and c-Met attenuated the invasion and migration of the PCa cells (Figs. 3C and $\mathrm{D}, 4 \mathrm{C}$ and $\mathrm{D}$ and $5 \mathrm{~A}$ and $\mathrm{B}$ ). However, knockdown of c-Met upregulated E-cadherin and downregulated $\mathrm{N}$-cadherin expression in both the PC3 and DU145 cell lines (Fig. 4B), even though the knockdown of RON did not produce the same effect (Fig. 3B). Activation of both RON with MSP and c-Met with HGF led to enhanced invasion of $\mathrm{PCa}$ cells in vitro (Figs. 3D and E, 4E and F and 5A and B). Similarly, activation of c-Met with HGF led to the upregulation of N-cadherin and downregulation of E-cadherin expression in $\mathrm{PCa}$, but activation of RON with MSP had no effect (Figs. 3C and 4D). Cell scattering is a hallmark of cell invasion and metastasis that consists of cell-cell dissociation, cell spreading and motility. To determine the effect of stimulating c-Met on scatter, we treated cells with HGF and found that this resulted in a change from compact colonies to scattered colonies (Fig. 4F and G). The above results indicate that the downregulation of RON and c-Met further inhibited the metastasis and reversal of the EMT of PCa cells to some extent through the HGF/c-Met signaling pathway. Prior studies showed that RON mediated the EMT in cancer cell lines via the RAS-ERK signaling pathway in Madin-Darby canine kidney (MDCK) epithelial cells $(24,25)$. However, we found that MSP/RON signaling is a significant contributor to invasiveness and metastasis, but not EMT, in prostate cancer, similar to the effects observed in breast, pancreatic and colon cancer. The molecular mechanisms of PCa metastasis are complicated and require further investigation.

We further screened for signaling pathways that were involved in the regulation of RON- and c-Met-mediated PCa metastasis. A previous study revealed that RON and c-Met are canonically mediated by ERK1/2 signaling (20). Therefore, we assessed the expression of active ERK1/2 upon RON and c-Met knockdown and found that silencing of RON and c-Met decreased the levels of p-ERK1/2 (Fig. 6A), while activation of RON and c-Met increased the levels of p-ERK1/2 (Fig. 6B). These findings were further validated by treating cells with U0126, which significantly suppressed the MSP- or HGF-induced migration and invasion of PC3 and DU145 cells (Fig. 6C and D). Taken together, these results demonstrated that RON and c-Met can promote PCa metastasis through the ERK1/2 pathway.

In conclusion, the present study indicated that RON and c-Met expression are upregulated in PCa tissues and this facilitates the metastasis of PCa cells via ERK1/2 signaling. To the best of our knowledge, this is the first study to demonstrate that foretinib has a marked antitumor metastatic effect on PCa cells. Although the underlying mechanism remains to be fully elucidated, this study suggests that targeting RON and c-Met may be a rational therapeutic strategy for suppressing the metastasis of $\mathrm{PCa}$, especially for $\mathrm{PCa}$ patients who have increased expression of RON and c-Met.

\section{Acknowledgements}

The present study was supported by grants from the National Natural Science Foundation of China (grant no. 81271917) and the Natural Science Foundation of Zhejiang Province (grant nos. LY14H200002, LY15H200002 and LY16H160023). We thank the Clinical Research Center from the Second Affiliated Hospital of Zhejiang University School of Medicine for essential technical support. We thank the American Journal Experts (AJE) for English language editing.

\section{References}

1. Siegel RL, Miller KD and Jemal A: Cancer statistics, 2015. CA Cancer J Clin 65: 5-29, 2015.

2. Park JC and Eisenberger MA: Advances in the treatment of metastatic prostate cancer. Mayo Clin Proc 90: 1719-1733, 2015.

3. Thiery JP, Acloque H, Huang RY and Nieto MA: Epithelialmesenchymal transitions in development and disease. Cell 139: 871-890, 2009.

4. Park M, Dean M, Kaul K, Braun MJ, Gonda MA and Vande Woude G: Sequence of MET protooncogene cDNA has features characteristic of the tyrosine kinase family of growthfactor receptors. Proc Natl Acad Sci USA 84: 6379-6383, 1987.

5. Chang K, Karnad A, Zhao S and Freeman JW: Roles of c-Met and RON kinases in tumor progression and their potential as therapeutic targets. Oncotarget 6: 3507-3518, 2015.

6. Ronsin C, Muscatelli F, Mattei MG and Breathnach R: A novel putative receptor protein tyrosine kinase of the met family. Oncogene 8: 1195-1202, 1993.

7. Birchmeier C, Birchmeier W, Gherardi E and Vande Woude GF: Met, metastasis, motility and more. Nat Rev Mol Cell Biol 4: 915-925, 2003.

8. Wang J, Rajput A, Kan JLC, Rose R, Liu XQ, Kuropatwinski K, Hauser J, Beko A, Dominquez I, Sharratt EA, et al: Knockdown of Ron kinase inhibits mutant phosphatidylinositol 3-kinase and reduces metastasis in human colon carcinoma. J Biol Chem 284: 10912-10922, 2009. 
9. Lee WY, Chen HH, Chow NH, Su WC, Lin PW and Guo HR: Prognostic significance of co-expression of RON and MET receptors in node-negative breast cancer patients. Clin Cancer Res 11: 2222-2228, 2005.

10. Faria CC, Golbourn BJ, Dubuc AM, Remke M, Diaz RJ, Agnihotri S, Luck A, Sabha N, Olsen S, Wu X, et al: Foretinib is effective therapy for metastatic sonic hedgehog medulloblastoma. Cancer Res 75: 134-146, 2015.

11. Kataoka Y, Mukohara T, Tomioka H, Funakoshi Y, Kiyota N Fujiwara Y, Yashiro M, Hirakawa K, Hirai M and Minami H: Foretinib (GSK1363089), a multi-kinase inhibitor of MET and VEGFRs, inhibits growth of gastric cancer cell lines by blocking inter-receptor tyrosine kinase networks. Invest New Drugs 30: $1352-1360,2012$

12. Huynh H, Ong R and Soo KC: Foretinib demonstrates anti-tumor activity and improves overall survival in preclinical models of hepatocellular carcinoma. Angiogenesis 15: 59-70, 2012.

13. You WK, Sennino B, Williamson CW, Falcón B, Hashizume H, Yao LC, Aftab DT and McDonald DM: VEGF and c-Met blockade amplify angiogenesis inhibition in pancreatic islet cancer. Cancer Res 71: 4758-4768, 2011.

14. Shah MA, Wainberg ZA, Catenacci DV, Hochster HS, Ford J, Kunz P, Lee FC, Kallender H, Cecchi F, Rabe DC, et al: Phase II study evaluating 2 dosing schedules of oral foretinib (GSK1363089), cMET/VEGFR2 inhibitor, in patients with metastatic gastric cancer. PLoS One 8: e54014, 2013.

15. Choueiri TK, Vaishampayan U, Rosenberg JE, Logan TF, Harzstark AL, Bukowski RM, Rini BI, Srinivas S, Stein MN, Adams LM, et al: Phase II and biomarker study of the dual MET/ VEGFR2 inhibitor foretinib in patients with papillary renal cell carcinoma. J Clin Oncol 31: 181-186, 2013.

16. Seiwert T, Sarantopoulos J, Kallender H, McCallum S, Keer HN and Blumenschein G Jr: Phase II trial of single-agent foretinib (GSK1363089) in patients with recurrent or metastatic squamous cell carcinoma of the head and neck. Invest New Drugs 31: 417-424, 2013.
17. Yau T, Yen CJ, Chen PJ, Chau Y, Lencioni R, Kallender H, Ottesen LH and Poon RTP: A phase I/II study of foretinib, an oral multikinase inhibitor targeting MET, RON, AXL, TIE-2, and VEGFR in advanced hepatocellular carcinoma (HCC). J Hepatol 54: S268, 2011.

18. Knubel KH, Pernu BM, Sufit A, Nelson S, Pierce AM and Keating AK: MerTK inhibition is a novel therapeutic approach for glioblastoma multiforme. Oncotarget 5: 1338-1351, 2014.

19. Shtutman M, Levina E, Ohouo P, Baig M and Roninson IB: Cell adhesion molecule L1 disrupts E-cadherin-containing adherens junctions and increases scattering and motility of MCF7 breast carcinoma cells. Cancer Res 66: 11370-11380, 2006.

20. Yao HP, Zhou YQ, Zhang R and Wang MH: MSP-RON signalling in cancer: Pathogenesis and therapeutic potential. Nat Rev Cancer 13: 466-481, 2013.

21. Kretschmann KL, Eyob H, Buys SS and Welm AL: The macrophage stimulating protein/Ron pathway as a potential therapeutic target to impede multiple mechanisms involved in breast cancer progression. Curr Drug Targets 11: 1157-1168, 2010.

22. Follenzi A, Bakovic S, Gual P, Stella MC, Longati P and Comoglio PM: Cross-talk between the proto-oncogenes Met and Ron. Oncogene 19: 3041-3049, 2000.

23. Benvenuti S, Lazzari L, Arnesano A, Li Chiavi G, Gentile A and Comoglio PM: Ron kinase transphosphorylation sustains MET oncogene addiction. Cancer Res 71: 1945-1955, 2011.

24. Wang D, Shen Q, Chen YQ and Wang MH: Collaborative activities of macrophage-stimulating protein and transforming growth factor-betal in induction of epithelial to mesenchymal transition: Roles of the RON receptor tyrosine kinase. Oncogene 23: 1668-1680, 2004.

25. Ma Q, Guin S, Padhye SS, Zhou YQ, Zhang RW and Wang MH: Ribosomal protein S6 kinase (RSK)-2 as a central effector molecule in RON receptor tyrosine kinase mediated epithelial to mesenchymal transition induced by macrophage-stimulating protein. Mol Cancer 10: 66, 2011. 